\title{
Verifying Wind Profile Equations Under Hurricane Conditions
}

\author{
S. A. Hsu*
}

Coastal Studies Institute, Louisiana State University, Baton Rouge, LA 70803

\begin{abstract}
During Hurricanes Gustav and Ike in 2008 simultaneous measurements of wind speed at $122 \mathrm{~m}$ and both wind speed and gust at $5 \mathrm{~m}$ as well as wave parameters were made at National Data Buoy Center Stations 42364 and 42040 , respectively, in the Gulf of Mexico. It is found that the wind speed at $122 \mathrm{~m}$ can be estimated satisfactory by either the logarithmic wind profile equation (using either roughness length or gust factor as the input) or the power-law wind profile equation using the exponent value of 0.10. It is recommended that for the wind load analysis at any height under hurricane conditions, the input for wind speed can be estimated by the standard buoy measurements at 5 or $10 \mathrm{~m}$ available routinely.
\end{abstract}

Keywords: Offshore wind profile, roughness length, friction velocity, hurricane winds and waves, hurricanes Gustav and Ike.

\section{INTRODUCTION}

During a storm, wind, wave and current loadings on offshore structures are all important. For many offshore wind load analysis, the wind speed at the elevation higher than the normal buoy anemometer height at either 5 or $10 \mathrm{~m}$ is required. During Hurricanes Gustav and Ike in 2008, simultaneous wind speed measurements at $122 \mathrm{~m}$ and both wind speed and gust at $5 \mathrm{~m}$ along with the wave parameters were made at the National Data Buoy Center (NDBC) Stations at 42364 and 42040, respectively, in the northeastern Gulf of Mexico (for station locations and hurricane tracks, see www.ndbc.noaa.gov). This unique data set is used in this study to evaluate or verify the wind profile equations.

\section{EQUATIONS}

Traditionally, both logarithmic and power-law wind profiles are used to estimate the wind speed variation with height. According to Hsu [1], during a storm, the familiar logarithmic wind profile is valid such that

$\mathrm{Uz}=\left(\mathrm{U}^{*} / \mathrm{k}\right)(\ln (\mathrm{Z} / \mathrm{Zo}))$

Where $\mathrm{Uz}_{\mathrm{z}}$ is the wind speed at height $\mathrm{Z}, \mathrm{U}^{*}$ is the friction velocity, $\mathrm{k}$ is the von Karman constant $(=0.4)$ and Zo is the roughness length.

Whereas the power-law equation states that (see, e.g. Hsu [1])

$\mathrm{Uz} / \mathrm{Uref}=(\mathrm{Z} / \mathrm{Zref})^{\wedge} \mathrm{p}$

For $\mathrm{Z}>\mathrm{Zref}$

Where Uref is the wind speed measurements at the anemometer or reference height, Zref, at either 5 or $10 \mathrm{~m}$ on the NDBC buoys and $\mathrm{p}$ is the exponent for the power-law wind profile.

Because Equation (1) has two unknown parameters (i.e., $\mathrm{U}^{*}$ and $\mathrm{Zo}$ ) in one equation and (2) requires the exponent

\footnotetext{
*Address correspondence to this author at the Coastal Studies Institute, Louisiana State University, Baton Rouge,LA 70803; Tel: 225-578-2962; Fax: 225-578-2520; E-mail: sahsu@1su.edu
}

from the literature, it is the purpose of this article to provide engineers with simplified equations to estimate offshore wind speed at any height for more accurate wind load analysis, since the inputs are based only upon both direct wind speed and gust or wave measurements from numerous NDBC buoys, which are available routinely.

\section{METHODS}

According to Equation (1), the difference in wind speed between level $\mathrm{Z}$ (higher elevation) and level Zref (lower elevation), i.e. by eliminating $U^{*}$, is

$\mathrm{Uz} / \mathrm{Uref}=\ln (\mathrm{Z} / \mathrm{Zo}) / \ln (\mathrm{Zref} / \mathrm{Zo})$

According to Taylor and Yelland [2], the roughness parameter $\mathrm{Zo}$ can be computed such that,

$\mathrm{Zo} / \mathrm{Hs}=1200 *(\mathrm{Hs} / \mathrm{Lp})^{\wedge} 4.5$

And, for deep water wave,

$\mathrm{Lp}=1.56^{*} \mathrm{Tp}^{\wedge} 2$

Where Hs and Lp are the significant wave height and peak wavelength for the combined sea and swell spectrum, and Tp is the corresponding wave period. Note that Hs is defined as the average height of the highest one-third of the waves observed at a specific point during the 20-minute sampling period.

According to Hsu [3, p.112], Equation (1) can also be used to estimate $\mathrm{Uz}$ from $\mathrm{U}^{*}$ by eliminating Zo such that

$\mathrm{Uz}-\mathrm{Uref}=\left(\mathrm{U}^{*} / \mathrm{k}\right) \ln (\mathrm{Z} / \mathrm{Zref})$

Furthermore, according to Hsu and Blanchard [4] that

$\mathrm{U}^{*}=0.2(\mathrm{U} 5$ gust $-\mathrm{U} 5)$

Where U5gust and U5 are the wind gust and mean wind speed measurements at $5 \mathrm{~m}$, respectively. All units of $\mathrm{U}^{*}$, U5gust and U5 are in $\mathrm{m} / \mathrm{s}$.

Now, if we set Uz and Uref for the wind speed measurements at $122 \mathrm{~m}$ and $5 \mathrm{~m}$, respectively, Equation (6) becomes

$\mathrm{U} 122=\mathrm{U} 5+8 \mathrm{U}^{*}$ 
Or, = U5 + 1.6 (U5gust - U5)

The exponent $\mathrm{p}(=0.10)$ in Equation (2) was measured by Hsu [3, p.202] under neutral stability conditions. Thus, Equation (2) can be reduced to

$\mathrm{Uz} / \mathrm{Uref}=(\mathrm{Z} / \mathrm{Zref})^{\wedge} 0.10$

Or, simply, by substituting $\mathrm{Uz}=\mathrm{U} 122$, Uref $=\mathrm{U} 5$, $\mathrm{Z}=122 \mathrm{~m}$ and

Table 1. Nearly Simultaneous Measurements of Wind Speed at $122 \mathrm{~m}$ at NDBC Station 42364 and Wind Speed and Gust at $5 \mathrm{~m}$ and Wave Parameters at Buoy 42040 During Gustav and Ike in 2008 in Gulf of Mexico

\begin{tabular}{|c|c|c|c|c|c|c|c|c|c|c|c|c|c|c|}
\hline 42364 & & & & Wind & U122m & 42040 & & & & Wind & U5 & G5 & Hs & $\mathbf{T p}$ \\
\hline Month & Day & Hour & Min. & Dir. & $\mathrm{m} / \mathrm{s}$ & Month & Day & Hour & Min. & Dir. & $\mathbf{m} / \mathbf{s}$ & $\mathbf{m} / \mathbf{s}$ & $\mathbf{m}$ & Sec. \\
\hline 8 & 31 & 2 & 0 & 70 & 9 & 8 & 31 & 1 & 50 & 76 & 6.6 & 7.5 & 0.6 & 4 \\
\hline 8 & 31 & 5 & 0 & 70 & 11 & 8 & 31 & 4 & 50 & 73 & 8.9 & 10.7 & 0.73 & 4.17 \\
\hline 8 & 31 & 6 & 0 & 70 & 13 & 8 & 31 & 5 & 50 & 69 & 8.5 & 9.8 & 0.85 & 4 \\
\hline 8 & 31 & 8 & 0 & 70 & 12 & 8 & 31 & 7 & 50 & 72 & 8.7 & 10.3 & 1.14 & 4.76 \\
\hline 8 & 31 & 13 & 0 & 50 & 13 & 8 & 31 & 12 & 50 & 51 & 10.9 & 13.4 & 1.76 & 6.67 \\
\hline 8 & 31 & 16 & 0 & 50 & 17 & 8 & 31 & 15 & 50 & 50 & 10.9 & 12.9 & 2.5 & 6.25 \\
\hline 8 & 31 & 19 & 0 & 60 & 15 & 8 & 31 & 18 & 50 & 48 & 12 & 15.2 & 2.82 & 7.14 \\
\hline 8 & 31 & 21 & 0 & 50 & 22 & 8 & 31 & 20 & 50 & 45 & 13.2 & 16.2 & 3.17 & 8.33 \\
\hline 8 & 31 & 22 & 0 & 60 & 18 & 8 & 31 & 21 & 50 & 59 & 15.3 & 19.6 & 3.54 & 7.69 \\
\hline 8 & 31 & 23 & 0 & 50 & 19 & 8 & 31 & 22 & 50 & 64 & 17 & 20.8 & 3.69 & 8.33 \\
\hline 9 & 1 & 0 & 0 & 50 & 21 & 8 & 31 & 23 & 50 & 54 & 18.7 & 23.6 & 5.79 & 16 \\
\hline 9 & 1 & 1 & 0 & 50 & 26 & 9 & 1 & 0 & 50 & 58 & 19.3 & 23.5 & 7.95 & 13.79 \\
\hline 9 & 1 & 2 & 0 & 40 & 28 & 9 & 1 & 1 & 50 & 48 & 18.8 & 22.7 & 7.8 & 13.79 \\
\hline 9 & 1 & 3 & 0 & 40 & 27 & 9 & 1 & 2 & 50 & 58 & 20.6 & 26.9 & 7.57 & 13.79 \\
\hline 9 & 1 & 4 & 0 & 70 & 31 & 9 & 1 & 3 & 50 & 60 & 20.6 & 25.4 & 7.77 & 12.12 \\
\hline 9 & 1 & 7 & 0 & 80 & 29 & 9 & 1 & 6 & 50 & 83 & 21.9 & 27.3 & 10.32 & 12.9 \\
\hline 9 & 1 & 8 & 0 & 110 & 32 & 9 & 1 & 7 & 50 & 91 & 21.7 & 27 & 9.67 & 11.43 \\
\hline 9 & 1 & 9 & 0 & 120 & 28 & 9 & 1 & 8 & 50 & 103 & 21.7 & 27.8 & 8.44 & 13.79 \\
\hline 9 & 1 & 10 & 0 & 130 & 28 & 9 & 1 & 9 & 50 & 116 & 20.7 & 25.5 & 8.2 & 12.9 \\
\hline 9 & 1 & 11 & 0 & 140 & 28 & 9 & 1 & 10 & 50 & 123 & 19.7 & 25 & 8.56 & 12.9 \\
\hline 9 & 1 & 12 & 0 & 150 & 24 & 9 & 1 & 11 & 50 & 136 & 21.6 & 27.2 & 7.9 & 11.43 \\
\hline 9 & 1 & 13 & 0 & 150 & 27 & 9 & 1 & 12 & 50 & 133 & 19.3 & 24 & 8.33 & 11.43 \\
\hline 9 & 1 & 17 & 0 & 160 & 20 & 9 & 1 & 16 & 50 & 141 & 17.1 & 22.2 & 5.62 & 11.43 \\
\hline 9 & 1 & 20 & 0 & 150 & 14 & 9 & 1 & 19 & 50 & 136 & 12 & 14.2 & 5.26 & 10 \\
\hline 9 & 2 & 3 & 0 & 140 & 16 & 9 & 2 & 2 & 50 & 135 & 12.6 & 15.6 & 3.75 & 9.09 \\
\hline 9 & 2 & 6 & 0 & 150 & 14 & 9 & 2 & 5 & 50 & 140 & 11.5 & 13.2 & 3.25 & 7.69 \\
\hline 9 & 11 & 7 & 0 & 70 & 17 & 9 & 11 & 6 & 50 & 91 & 10.5 & 14.1 & 6.43 & 14.81 \\
\hline 9 & 11 & 9 & 0 & 60 & 21 & 9 & 11 & 8 & 50 & 69 & 12.4 & 15.2 & 7.33 & 14.81 \\
\hline 9 & 11 & 10 & 0 & 70 & 19 & 9 & 11 & 9 & 50 & 65 & 11 & 13.1 & 7.54 & 14.81 \\
\hline
\end{tabular}

Zref $=5 \mathrm{~m}$, we have

$\mathrm{U} 122=1.38 \mathrm{U} 5$

Our purpose is to verify all foregoing equations against the measurements as listed in Table 1. Note that from August 31 thru September 2, 2008 our stations were affected by Gustav and from September 11 thru 12 by Ike. 
Table. 1. Cont....

\begin{tabular}{|c|c|c|c|c|c|c|c|c|c|c|c|c|c|c|}
\hline 9 & 11 & 12 & 0 & 80 & 22 & 9 & 11 & 11 & 50 & 79 & 17.4 & 21 & 7.58 & 11.43 \\
\hline 9 & 11 & 13 & 0 & 70 & 19 & 9 & 11 & 12 & 50 & 79 & 16.8 & 21.5 & 7.61 & 14.81 \\
\hline 9 & 11 & 14 & 0 & 70 & 20 & 9 & 11 & 13 & 50 & 84 & 15.4 & 18.8 & 7.59 & 14.81 \\
\hline 9 & 11 & 15 & 0 & 80 & 21 & 9 & 11 & 14 & 50 & 83 & 15.8 & 19.4 & 8.6 & 12.9 \\
\hline 9 & 11 & 16 & 0 & 70 & 22 & 9 & 11 & 15 & 50 & 84 & 15.9 & 19.4 & 8.2 & 12.9 \\
\hline 9 & 11 & 17 & 0 & 80 & 25 & 9 & 11 & 16 & 50 & 81 & 15.8 & 19.3 & 7.56 & 14.81 \\
\hline 9 & 11 & 18 & 0 & 80 & 23 & 9 & 11 & 17 & 50 & 82 & 17.2 & 21.9 & 7.61 & 14.81 \\
\hline 9 & 11 & 19 & 0 & 70 & 21 & 9 & 11 & 18 & 50 & 80 & 16.4 & 19.9 & 7.75 & 13.79 \\
\hline 9 & 11 & 20 & 0 & 90 & 20 & 9 & 11 & 19 & 50 & 84 & 16.9 & 21.2 & 7.79 & 13.79 \\
\hline 9 & 11 & 21 & 0 & 90 & 20 & 9 & 11 & 20 & 50 & 84 & 16.2 & 20.2 & 6.99 & 14.81 \\
\hline 9 & 11 & 22 & 0 & 80 & 23 & 9 & 11 & 21 & 50 & 89 & 15.5 & 20.2 & 8.23 & 13.79 \\
\hline 9 & 11 & 23 & 0 & 80 & 23 & 9 & 11 & 22 & 50 & 90 & 16 & 20 & 7.73 & 13.79 \\
\hline 9 & 12 & 0 & 0 & 80 & 18 & 9 & 11 & 23 & 50 & 87 & 15.2 & 17.7 & 8.03 & 14.81 \\
\hline 9 & 12 & 1 & 0 & 100 & 22 & 9 & 12 & 0 & 50 & 89 & 16.3 & 19.9 & 7.75 & 13.79 \\
\hline 9 & 12 & 3 & 0 & 110 & 24 & 9 & 12 & 2 & 50 & 94 & 15.5 & 22.5 & 7.07 & 14.81 \\
\hline 9 & 12 & 4 & 0 & 100 & 18 & 9 & 12 & 3 & 50 & 97 & 15.3 & 19.5 & 6.88 & 13.79 \\
\hline 9 & 12 & 5 & 0 & 110 & 24 & 9 & 12 & 4 & 50 & 97 & 15.5 & 19.9 & 7.08 & 13.79 \\
\hline 9 & 12 & 6 & 0 & 100 & 22 & 9 & 12 & 5 & 50 & 105 & 15.6 & 19.8 & 6.4 & 13.79 \\
\hline 9 & 12 & 7 & 0 & 110 & 23 & 9 & 12 & 6 & 50 & 104 & 16.4 & 20.2 & 6.41 & 13.79 \\
\hline 9 & 12 & 8 & 0 & 90 & 19 & 9 & 12 & 7 & 50 & 105 & 15.6 & 19.8 & 7.2 & 12.9 \\
\hline 9 & 12 & 9 & 0 & 110 & 19 & 9 & 12 & 8 & 50 & 107 & 15.1 & 19.8 & 5.72 & 12.9 \\
\hline 9 & 12 & 10 & 0 & 110 & 22 & 9 & 12 & 9 & 50 & 107 & 16 & 20.3 & 6.09 & 12.9 \\
\hline 9 & 12 & 11 & 0 & 110 & 19 & 9 & 12 & 10 & 50 & 107 & 16.4 & 21.4 & 6.52 & 12.9 \\
\hline 9 & 12 & 12 & 0 & 120 & 21 & 9 & 12 & 11 & 50 & 111 & 16.1 & 20.9 & 6.4 & 12.12 \\
\hline 9 & 12 & 13 & 0 & 120 & 22 & 9 & 12 & 12 & 50 & 115 & 17.1 & 20.1 & 5.74 & 12.12 \\
\hline 9 & 12 & 14 & 0 & 120 & 18 & 9 & 12 & 13 & 50 & 113 & 15.6 & 19.3 & 5.47 & 12.12 \\
\hline 9 & 12 & 15 & 0 & 130 & 20 & 9 & 12 & 14 & 50 & 117 & 15.1 & 18.1 & 5.63 & 12.12 \\
\hline 9 & 12 & 16 & 0 & 130 & 17 & 9 & 12 & 15 & 50 & 118 & 14.8 & 18.1 & 5.5 & 12.12 \\
\hline 9 & 12 & 17 & 0 & 130 & 18 & 9 & 12 & 16 & 50 & 121 & 14.2 & 17.6 & 5.38 & 11.43 \\
\hline 9 & 12 & 19 & 0 & 140 & 16 & 9 & 12 & 18 & 50 & 130 & 13.5 & 17.3 & 5.36 & 11.43 \\
\hline 9 & 12 & 21 & 0 & 130 & 18 & 9 & 12 & 20 & 50 & 127 & 12.3 & 15 & 5.05 & 10.81 \\
\hline 9 & 12 & 22 & 0 & 140 & 17 & 9 & 12 & 21 & 50 & 124 & 13.3 & 16.2 & 5.03 & 11.43 \\
\hline 9 & 13 & 1 & 0 & 130 & 19 & 9 & 13 & 0 & 50 & 124 & 12.8 & 15.9 & 4.81 & 12.9 \\
\hline 9 & 13 & 2 & 0 & 130 & 17 & 9 & 13 & 1 & 50 & 123 & 12.5 & 15.4 & 4.87 & 12.9 \\
\hline 9 & 13 & 3 & 0 & 130 & 16 & 9 & 13 & 2 & 50 & 125 & 12 & 14.6 & 4.26 & 10.81 \\
\hline 9 & 13 & 4 & 0 & 140 & 15 & 9 & 13 & 3 & 50 & 125 & 12.7 & 14.8 & 5.1 & 12.9 \\
\hline 9 & 13 & 5 & 0 & 140 & 16 & 9 & 13 & 4 & 50 & 125 & 12.4 & 14.9 & 4.76 & 12.12 \\
\hline 9 & 13 & 7 & 0 & 150 & 13 & 9 & 13 & 6 & 50 & 133 & 10.9 & 13.4 & 4.7 & 12.12 \\
\hline
\end{tabular}


Table. 1. Cont....

\begin{tabular}{|c|c|c|c|c|c|c|c|c|c|c|c|c|c|c|}
\hline 9 & 13 & 8 & 15 & 140 & 15 & 9 & 13 & 7 & 50 & 129 & 12.1 & 14.5 & 4.52 & 11.43 \\
\hline 9 & 13 & 9 & 0 & 140 & 14 & 9 & 13 & 8 & 50 & 133 & 11.5 & 13.9 & 4.05 & 10.81 \\
\hline 9 & 13 & 10 & 0 & 150 & 14 & 9 & 13 & 9 & 50 & 134 & 10.9 & 13 & 4.23 & 11.43 \\
\hline 9 & 13 & 12 & 0 & 140 & 15 & 9 & 13 & 11 & 50 & 134 & 10.1 & 13 & 3.59 & 11.43 \\
\hline 9 & 13 & 17 & 0 & 160 & 12 & 9 & 13 & 16 & 50 & 144 & 9.8 & 11.3 & 2.58 & 10.81 \\
\hline 9 & 13 & 19 & 0 & 150 & 12 & 9 & 13 & 18 & 50 & 144 & 9.3 & 11.2 & 2.62 & 10.81 \\
\hline 9 & 14 & 0 & 0 & 150 & 11 & 9 & 13 & 23 & 50 & 141 & 8.5 & 10.7 & 2.39 & 10 \\
\hline 9 & 14 & 1 & 0 & 150 & 11 & 9 & 14 & 0 & 50 & 143 & 8.6 & 9.9 & 2.46 & 7.69 \\
\hline 9 & 14 & 3 & 0 & 160 & 11 & 9 & 14 & 2 & 50 & 146 & 8.8 & 10.6 & 2.38 & 10 \\
\hline 9 & 14 & 12 & 0 & 180 & 10 & 9 & 14 & 11 & 50 & 173 & 7.2 & 8.6 & 1.89 & 7.14 \\
\hline
\end{tabular}

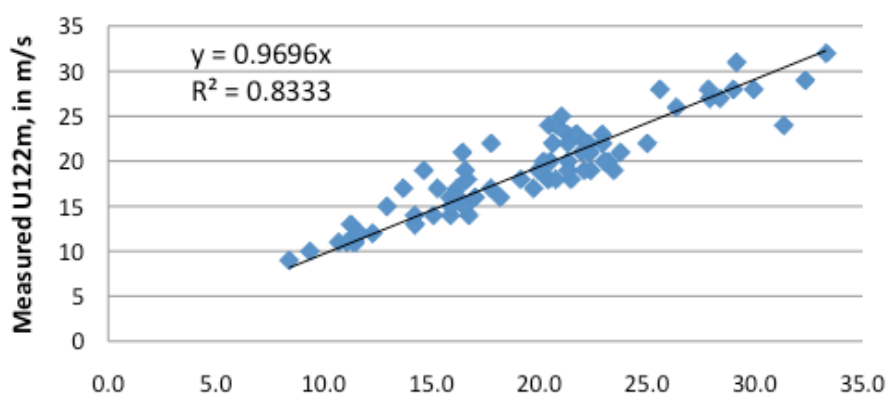

Estimated $U 122 \mathrm{~m}$, in $\mathrm{m} / \mathrm{s}$, based on Zo formulation

Fig. (1). A comparison between estimated and measured wind speed at $122 \mathrm{~m}$ based on Zo formulation.

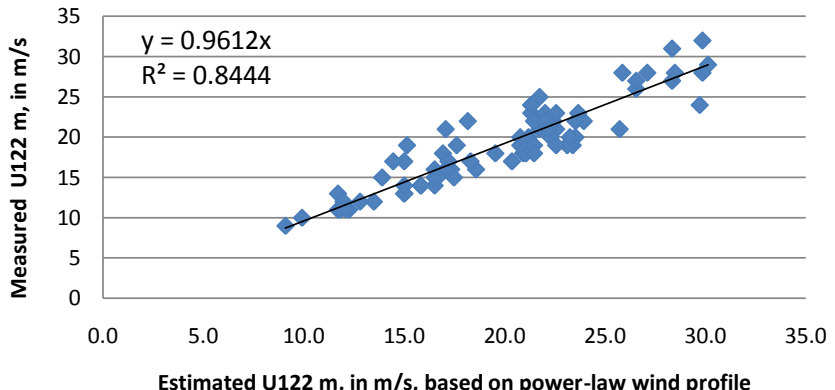

Fig. (2). A comparison between estimated and measured wind speed at $122 \mathrm{~m}$ based on the power-law wind profile.

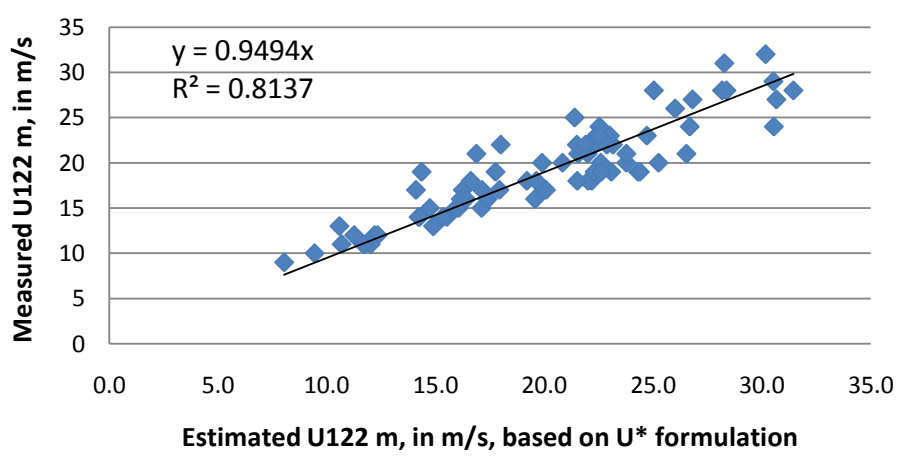

Fig. (3). A comparison between estimated and measured wind speed at $122 \mathrm{~m}$ based on $\mathrm{U}^{*}$ formulation.

\section{RESULTS}

Our results are presented in Figs. (1 to 3). Note that for better comparison the intercept in the linear regression is imposed to equal to zero. Now, if one accepts these high $\mathrm{R}^{2}$ values, then, the foregoing wind profile equations are verified. Note that according to NDBC, the margin of error in the wind speed measurement is approximately plus or 
minus10 \%. Since the slopes in these Figures are close to one, which are within the $10 \%$ margin of errors, it is recommended that these equations be employed operationally using only the direct measurements of both wind speed and gust or wave parameters from nearby NDBC buoy stations. It is surprising that the exponent in Equation $(9)$, i.e. p $(=0.10)$, works also well under hurricane conditions.

\section{CONCLUSIONS}

On the bases of simultaneous measurements of wind speed at $122 \mathrm{~m}$ and both wind speed and gust at $5 \mathrm{~m}$ along with the wave parameters in the Gulf of Mexico during Hurricanes Gustav and Ike in 2008, both logarithmic and powerlaw wind profile equations are verified. These equations can be used to estimate the wind speed at any height for wind load analysis under hurricane conditions using the direct buoy measurements which are available routinely. If the buoy data are not available, one can set the exponent, $\mathrm{p}=0.10$, in the power-law wind profile equation.

\section{REFERENCES}

[1] S.A. Hsu, "Estimating overwater friction velocity and exponent of Power-law wind profile from gust factor during storms", J. Waterways, Port, Coast. Ocean Eng., vol. 129, no. 4, pp. 174-177, 2003.

[2] P.K. Taylor, and M.J. Yelland, "The dependence of sea surface Roughness on the height and steepness of the waves", J. Phys. Oceanogr., vol. 31, pp. 572-590, 2001.

[3] S.A. Hsu, Coastal Meteorology. Academic Press, USA, 1988.

[4] S.A. Hsu, and B.W. Blanchard, "Estimating overwater turbulence Intensity from routine gust-factor measurements", J. Appl. Meteorol., vol. 43, no. 12, pp.1911-1916, 2004.

Received: February 9, 2011

Revised: March 31, 2011

Accepted: April 5, 2011

(c) S. A. Hsu; Licensee Bentham Open.

This is an open access article licensed under the terms of the Creative Commons Attribution Non-Commercial License (http://creativecommons.org/licenses/by-nc/3.0/) which permits unrestricted, non-commercial use, distribution and reproduction in any medium, provided the work is properly cited. 